\title{
La salud mental de los enfermeros en un hospital andino en tiempos de COVID-19
}

\author{
The mental health of nurses in an Andean hospital in times of COVID-19 \\ A saúde mental das enfermeiras em um hospital andino na época do COVID-19
}

Llerme Núñez Zarazu

opebri@yahoo.com

https://orcid.org/0000-0002-8011-5072

Veronica Shirley Alberto Veramendi

vero_shirley03@yahoo.es

https://orcid.org/0000-0002-1578-9417

Silvia Elizabet Reyes Narváez

reynaelizabet26@hotmail.com

https://orcid.org/0000-0002-9624-1997

\author{
Nalda Soraya Núñez Zarazu \\ soralina24@gmail.com \\ https://orcid.org/0000-0002-6389-4609
}

Edwin Johny Asnate Salazar

edwin_johny@hotmail.com, edwin.johny@gmail.com https://orcid.org/0000-0002-4319-8964

Arturo Robinson Amado Oncoy
thecharmedoflove@hotmail.com
https://orcid.org/0000-0002-8982-6700

Universidad Nacional Santiago Antúnez de Mayolo, Huaraz-Perú

Recibido 7 de junio 2021 | Arbitrado y aceptado 22 de julio 2021 | Publicado en 16 de agosto 2021

\section{RESUMEN}

La investigación tuvo como objetivo comparar la salud mental de los enfermeros que laboraban en el área COVID-19 respecto a otras áreas laborales en un hospital público de Huaraz. Materiales y Métodos. Fue descriptivo, transversal y correlacional. La muestra intencional estuvo conformada por los 110 profesionales de enfermería que aceptaron responder al cuestionario online del DASS-21 y Escala de Athenas. Resultados niveles sin diferencia significativa de ansiedad, insomnio, depresión y estrés en los enfermeros que laboran en áreas de atención de pacientes con COVID-19 y otras. La depresión estuvo asociada a la convivencia con personas vulnerables en el hogar y al diagnóstico positivo a enfermedades de salud mental antes de la pandemia. La ansiedad estuvo asociada a la convivencia con familiares, a la práctica de alguna religión y al diagnóstico personal positivo a COVID-19. El estrés estuvo asociada al consumo de psicofármacos. Conclusiones. Los resultados indican que se requieren intervenciones de salud mental dirigidos al personal de enfermería que labora en todo el hospital en el contexto de la pandemia por coronavirus.

Palabras clave: Enfermería; COVID-19; salud mental

\begin{abstract}
The objective of the research was to compare the mental health of nurses working in the COVID-19 area with respect to other work areas in a public hospital in Huaraz. Materials and Methods. It was descriptive, cross-sectional and correlational. The purposive sample consisted of 110 nursing professionals who agreed to answer the DASS-21 and Athens Scale online questionnaire. The results showed no significant difference in the levels of anxiety, insomnia, depression and stress in nurses working in patient care areas with COVID-19 and others. Depression was associated with living with vulnerable people at home and positive diagnosis of mental health illnesses before the pandemic. Anxiety was associated with living with family members, the practice of a religion, and a positive personal diagnosis of COVID19. Stress was associated with psychotropic drug use. Conclusions. The results indicate that mental health interventions are needed for nurses working throughout the hospital in the context of the coronavirus pandemic.
\end{abstract}

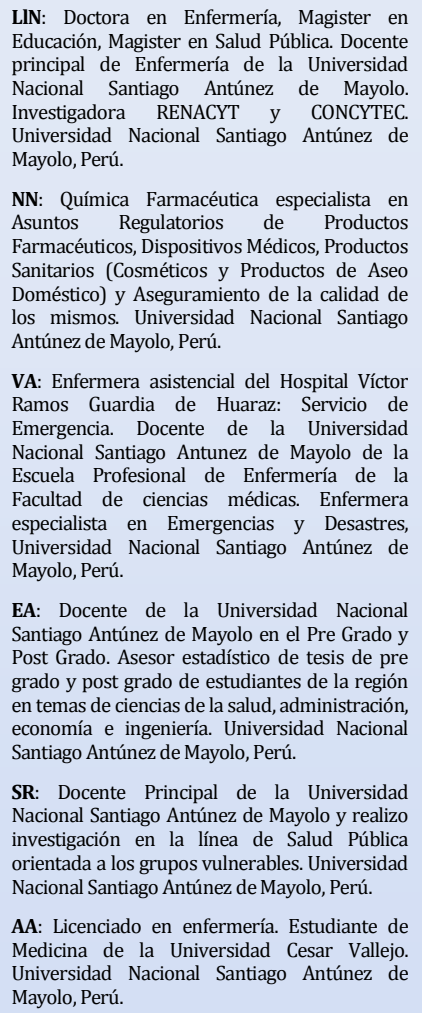
LIN: Doctora en Enfermería, Magister en
Educación, Magister en Salud Pública. Docente principal de Enfermería de la Universidad principal de Enfermería de la Universidad
Nacional Santiago Antúnez de Mayolo. Nacional Santiago Antúnez de Mayolo.
Investigadora RENACYT y CONCYTEC. Universidad Nacional Santiago Antúnez de Mayolo, Perú.

NN: Química Farmacéutica especialista en Asuntos Regulatorios de Productos Farmacéuticos, Dispositivos Médicos, Productos Sanitarios (Cosméticos y Productos de Aseo Doméstico) y Aseguramiento de la calidad de Doméstico) y Aseguramiento de la calidad de
los mismos. Universidad Nacional Santiago Antúnez de Mayolo, Perú.

VA: Enfermera asistencial del Hospital Víctor Ramos Guardia de Huaraz: Servicio de Emergencia. Docente de la Universidad Nacional Santiago Antunez de Mayolo de la Escuela Profesional de Enfermería de la Facultad de ciencias médicas. Enfermera especialista en Emergencias y Desastres, Universidad Nacional Santiago Antúnez de Mayolo, Perú.

EA: Docente de la Universidad Nacional Santiago Antúnez de Mayolo en el Pre Grado y Post Grado. Asesor estadístico de tesis de pre grado y post grado de estudiantes de la región en temas de ciencias de la salud, administración, economía e ingeniería. Universidad Nacional Santiago Antúnez de Mayolo, Perú

SR: Docente Principal de la Universidad Nacional Santiago Antúnez de Mayolo y realizo investigación en la línea de Salud Pública orientada a los grupos vulnerables. Universid Nacional Santiago Antúnez de Mayolo, Perú. Medicina de la Universidad Cesar Vallejo. Universidad Nacional Santiago Antúnez de Mayolo, Perú. 
LIN: Doctora en Enfermería, Magister en Educación, Magister en Salud Pública. Docente principal de Enfermería de la Universidad Nacional Santiago Antúnez de Mayolo. Nacional Santiago Antunez de Mayolo, Universidad Nacional Santiago Antúnez de Mayolo, Perú.

NN: Química Farmacéutica especialista en Asuntos Regulatorios de Productos Farmacéuticos, Dispositivos Médicos, Productos Sanitarios (Cosméticos y Productos de Aseo Samíśtico) y Aseguramiento de la calide Doméstico) y Aseguramiento de la calidad de Antúnez de Mayolo, Perú.

VA: Enfermera asistencial del Hospital Víctor Ramos Guardia de Huaraz: Servicio de Emergencia. Docente de la Universidad Nacional Santiago Antunez de Mayolo de la Escuela Profesional de Enfermería de la Facultad de ciencias médicas. Enfermera Facultid de cincias medicas. Enermera especialista en Energencias y Desastres, Mayolo, Perú.

EA: Docente de la Universidad Nacional Santiago Antúnez de Mayolo en el Pre Grado y Post Grado. Asesor estadístico de tesis de pre grado y post grado de estudiantes de la región grado y post grado de estudiantes de la región en temas de ciencias de la salud, administración Santiago Antúnez de Mayolo, Perú.

SR: Docente Principal de la Universidad Nacional Santiago Antúnez de Mayolo y realizo investigación en la línea de Salud Públic orientada a los grupos vulnerables. Universidad Nacional Santiago Antúnez de Mayolo, Perú.

AA: Licenciado en enfermería. Estudiante de Medicina de la Universidad Cesar Vallejo. Universidad Nacional Santiago Antúnez de Mayolo, Perú.

\begin{abstract}
RESUMO
A pesquisa teve como objetivo comparar a saúde mental dos enfermeiros que atuavam na área do COVID-19 em relação às demais áreas de atuação de um hospital público de Huaraz. Materiais e métodos. Foi descritivo, transversal e correlacional. A amostra intencional foi composta por 110 profissionais de enfermagem que concordaram em responder ao questionário online DASS21 e Escala de Athenas. Resultados. Níveis sem diferença significativa de ansiedade, insônia, depressão e estresse em enfermeiros que atuam em áreas de cuidado a pacientes com COVID-19 e outros. A depressão estava associada a viver com pessoas vulneráveis em casa e a um diagnóstico positivo de doenças mentais antes da pandemia. An ansiedade foi associada a viver com familiares, praticar uma religião e um diagnóstico pessoal positivo de COVID19. 0 estresse foi associado ao consumo de psicofármacos. Conclusões. Os resultados indicam que as intervenções em saúde mental são necessárias para a equipe de enfermagem que trabalha em todo o hospital no contexto da pandemia do coronavirus.
\end{abstract}

Palavras-chave: Enfermagem; COVID-19; saúde mental

\section{INTRODUCCIÓN}

A ctualmente existe una nueva crisis de salud pública que amenaza al mundo con la aparición y propagación del nuevo coronavirus del síndrome respiratorio agudo severo (SARS-CoV-2) (1). A medida que la pandemia por COVID-19 avanza, se aceleran los sistemas mundiales de atención médica, además estos se han visto abrumados por la carga de pacientes (2), lo que lleva a una gran presión psicológica por parte de los profesionales de enfermería que brindan atención en la unidad de cuidados críticos (3).

El profesional de enfermería tiene como principal característica la gestión del cuidado, es decir conservar la vida asegurando la satisfacción de las necesidades (4).

En Singapur e India encontraron que de 906 profesionales de salud que participaron en la encuesta, el 5,3\% presentaron depresión moderada a muy severa, el $8,7 \%$ ansiedad moderada a extremadamente severa, el $2,2 \%$ moderada a extremadamente estrés severo, y el 3,8\% niveles moderados a severos de angustia psicológica (5).

En Italia, hallaron en un estudio a nivel nacional sobre salud mental entre los trabajadores de atención médica de primera línea y de segunda línea durante la pandemia por coronavirus, que, de un total de 681 encuestados, el 49,38\% padecieron estrés postraumático; el 24,73\% síntomas de depresión; el 19,80\% 
síntomas de ansiedad; el 8,27\% insomnio; y el $21,90 \%$ estrés percibido alto (6).

Muchos factores están contribuyendo a la angustia psicológica de enfermeros, y otros trabajadores de la salud que brindan atención directa de primera línea a pacientes con COVID-19, como los siguientes: tensión emocional y agotamiento físico, cuidado de compañeros de trabajo que pueden enfermarse gravemente, escasez de equipos de protección personal, preocupaciones acerca de infectar a miembros de la familia, escasez de ventiladores y otros equipos médicos cruciales, ansiedad por asumir roles clínicos nuevos o desconocidos y acceso limitado a servicios de salud mental para controlar la depresión, la ansiedad y la angustia psicológica $(7,8)$.

En Jordania, se reportó que el 21,5\% de los profesionales de salud presentó ansiedad, esto indicando que, durante los brotes de la pandemia, las personas se someten a condiciones extremadamente estresantes, lo que resulta un mayor riesgo de desarrollar ansiedad y depresión (9). En Wuhan - China en el Hospital Wuhan Pulmonary, los enfermeros de primera línea que laboran en la unidad de cuidados intensivos experimentaban una gran carga de trabajo, fatiga a largo plazo, amenaza de infección y frustración con la muerte de los pacientes a quienes cuidan. También enfrentan ansiedad o incluso malentendidos entre los pacientes y sus familiares, además se reportó que, de 85 enfermeros, el $59 \%$ presentaba disminución del apetito o indigestión, el $55 \%$ refirió fatiga, el $45 \%$ manifestó dificultad para dormir, el $28 \%$ nerviosismo, el $26 \%$ llanto frecuente y el $2 \%$ pensamientos suicidas, los enfermeros jóvenes sin experiencia en el cuidado de pacientes críticos se enfrentan a una crisis psicológica mayor (3).

En España, se realizó un meta análisis sobre el impacto psicológico de la cuarentena en los trabajadores de la salud, donde se concluyó que los profesionales de la salud experimentan estrés agudo, síntomas depresivos, estrés postraumático y alcoholismo crónico (10).

Mientras que en Cuzco-Perú, se observó que el profesional de enfermería, que estaba en contacto directo con pacientes diagnósticos por COVID-19, tuvieron una prevalencia de depresión del $30,8 \%$ y ansiedad en un $41,8 \%$ y estrés de $34,1 \%$; además la relación con el afrontamiento de evitación podría generar problemas posteriores con la salud del personal (11).

La investigación tuvo como objetivos comparar la salud mental (ansiedad, depresión, estrés e insomnio) de los enfermeros que laboraban en el área COVID-19 respecto a otras áreas $\mathrm{y}$ determinar la relación de estos problemas de salud mental con factores de salud, familiares y sociodemográficos.

\section{MATERIALES Y MÉTODOS}

$\mathrm{L}$ a investigación tuvo un enfoque cuantitativo, descriptivo correlacional. La población estuvo conformada por todos los profesionales de enfermería que laboraban en el Hospital Víctor Ramos Guardia, según registros de recursos humanos a la fecha se encontraron trabajando un total de 170 profesionales de enfermería, de los cuales 110 sólo aceptaron llenar la encuesta, por lo que constituyó la muestra de estudio.

Los criterios de inclusión fueron Profesional de enfermería que labore en el Hospital Víctor Ramos Guardia, Profesional 
de enfermería de ambos sexos, Profesional de enfermería que labore en el área COVID-19 y no área COVID-19, Profesional de enfermería nombrado o contratado y Profesional de enfermería que acepte participar del estudio y firme el consentimiento informado.

Los criterios de exclusión fueron Profesional de enfermería que se encuentre de vacaciones o licencia de salud y los que no aceptaron el consentimiento informado.

La unidad de análisis estuvo constituida por cada personal profesional de enfermería. La muestra fue tomada de manera no aleatorizada y por conveniencia y se conformó por todos los profesionales que aceptaron responder la encuesta en línea y quedó conformada por 110 unidades de análisis: 51 enfermeros del área no COVID-19 y 59 enfermeros que laboraban en área COVID-19.

Se empleó como técnica la encuesta y como instrumento el cuestionario auto informado aplicado en línea. El cuestionario estuvo conformado por 45 ítems, de los cuales 16 ítems son preguntas generales, y 29 ítems son preguntas del estudio: 8 corresponden a la Escala de Athenas y 21 a la escala del DASS-21, ambas con validez y fiabilidad en el idioma español determinados por Portocarrero (12) y Ruiz (13) respectivamente.

La escala DASS-21, consta de 7 ítems para depresión, 7 ítems para ansiedad y 7 ítems para estrés, estas preguntas contienen respuestas tipo Likert (nunca, a veces, con frecuencia y casi siempre). Por otro lado, el EAI contiene ocho ítems, cada uno con respuestas tipo Likert con puntuaciones entre 0 y 3 . En la investigación obtuvieron una fiabilidad de 0,93 y 0,88 respectivamente.

Antes de la ejecución del estudió, se aplicó el instrumento a una muestra piloto, lo cual permitió afinar la comprensión léxica y además permitió obtener datos para realizar la prueba de confiabilidad a través del Alfa de Cronbach.

Los datos fueron procesados a través del software estadístico gratuito R Studio, el cual permitió realizar un análisis de normalidad de los datos y el análisis inferencial de regresión logística.

\section{RESULTADOS}

$4 \begin{aligned} & \text { continuación se muestran los } \\ & \text { resultados más } \\ & \text { obtenidos mediante la aplicación de }\end{aligned}$ los instrumentos relacionados con depresión y ansiedad en donde se observó que el $76,4 \%$ del total de profesionales de enfermería son mujeres. El $61 \%$ del total de enfermeros del área COVID-19 tienen edades mayores a 30 años, además se aprecia que $6,8 \%$ del total de enfermeros tiene alguna enfermedad crónica. Los enfermeros que trabajan en el área COVID19 son solteras (52,5\%). Además, se aprecia que el $72.9 \%$ del total de los enfermeros no participa en ninguna religión.

De los enfermeros que trabaja en el área COVID-19: el 59,3\% del total de enfermeros vive con niños o personas mayores de 65 años o con alguna enfermedad, el 81,4\% viven con su familia, la mayoría de estos profesionales laboran menos de 10 años y tienen miedo a contagiar con el COVID-19 a sus familiares. Además, el $54.2 \%$ manifiestan que trabajan con EPP incompletos. También afirman que alguien en su círculo social fuera de su hogar ha tenido COVID-19, además manifiesta alguien fuera de su hogar falleció con el COVID-19 y el 32.2\% ha sido diagnosticado con el COVID-19.

En la Tabla 1 se observa la distribución de la salud mental de los 
enfermeros del estudio, donde se pudo evidencia que el $32.2 \%$ que labora en el área no COVID-19 presentó insomnio, mientras que el 43.1\% del área COVID-19 padecen insomnio.

Tabla 1. Salud mental de enfermeros, Hospital Huaraz-Perú.

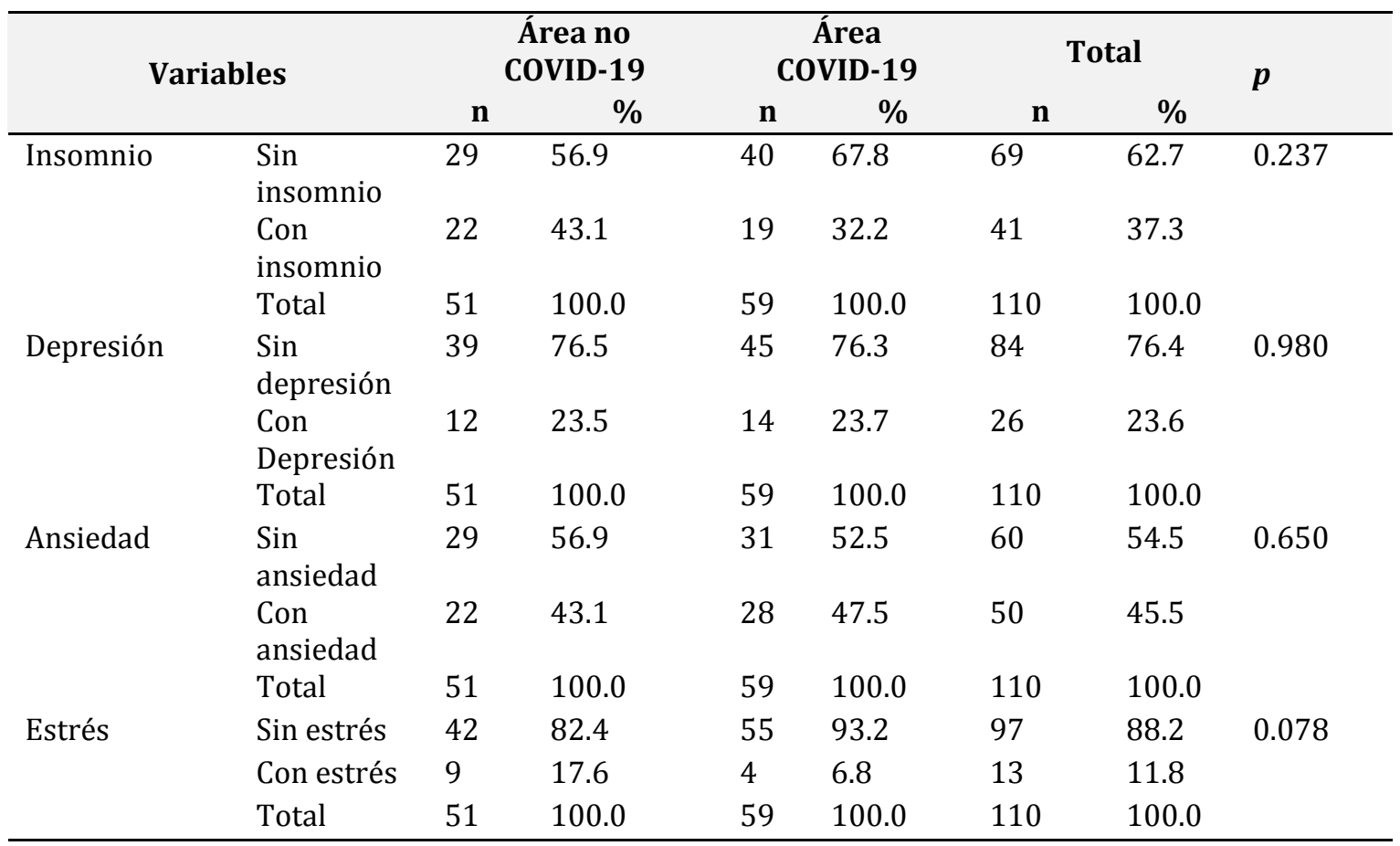

En cuanto a la depresión se pudo encontrar que el $23.7 \%$ que laboran en el área COVID-19 se sienten deprimidas, mientras que un $23.5 \%$ del total de enfermeros que trabajan en el área no COVID-19 también se deprimen. El 47.5\% del total de enfermeros que trabajan en el área COVID-19 tienen ansiedad, y en el área no COVID-19 el 43.1\%.

En cuanto al estrés se determinó que el 6.8\% de enfermeros que trabajan en el área COVID-19 tienen estrés, mientras que el $17.6 \%$ lo presentan en el área no COVID19.

Realizando una prueba de diferencias de proporciones usando la prueba chi cuadrado a una confianza de 95\%; no se halló diferencias significativas de los cuatro problemas de salud mental en los enfermeros en el área COVID-19 y área no COVID-19.

En la Tabla 2 se aprecia que, en la depresión de los enfermeros, que tuvieron en común ser diagnosticados con alguna enfermedad mental antes de la pandemia, para los que trabajan en el área no COVID19 fue de $16.7 \%$ siendo esta relación significativa $(\mathrm{p}<0,009)$. Para el grupo de enfermeros que trabajaron en el área COVID-19 y vivían con niños o personas mayores de 65 años o con alguna enfermedad, resultando también esta relación significativa. 
Tabla 2. Depresión en enfermeros de área COVID-19 y No COVID-19 según algunas variables, Hospital Huaraz-Perú.

\begin{tabular}{|c|c|c|c|c|c|c|c|c|}
\hline \multirow{2}{*}{ Variables } & & \multicolumn{2}{|c|}{ Sin depresión } & \multicolumn{2}{|c|}{ Con Depresión } & \multicolumn{2}{|c|}{ Total } & \multirow{2}{*}{$p$} \\
\hline & & $\mathbf{n}$ & $\%$ & $\mathbf{n}$ & $\%$ & $\mathbf{n}$ & $\%$ & \\
\hline \multicolumn{9}{|l|}{ AREA NO COVID-19 } \\
\hline \multirow{2}{*}{$\begin{array}{l}\text { Problema de salud } \\
\text { mental antes de la } \\
\text { pandemia }\end{array}$} & No & 39 & 100 & 10 & 83.3 & 49 & 96.1 & \multirow[t]{2}{*}{0,009} \\
\hline & $\mathrm{Si}$ & 0 & 0 & 2 & 16.7 & 2 & 3.9 & \\
\hline \multicolumn{9}{|l|}{ ÁREA COVID-19 } \\
\hline \multirow{2}{*}{$\begin{array}{l}\text { Hogar con personas } \\
\text { vulnerables }\end{array}$} & No & 15 & 33.3 & 9 & 64.3 & 24 & 40.7 & \multirow[t]{2}{*}{0,039} \\
\hline & $\mathrm{Si}$ & 30 & 66.7 & 5 & 35.7 & 35 & 59.3 & \\
\hline
\end{tabular}

En la Tabla 3 se presenta los enfermeros que trabajan en el área no COVID-19 que consumen algún medicamento tipo antidepresivo o ansiolítico, se relaciona significativamente con el estrés $(22.3 \%)$ y con el insomnio (13.6\%).

Tabla 3. Estrés e Insomnio en enfermeros en áreas No COVID_19 según algunas variables, Hospital Huaraz-Perú.

\begin{tabular}{|c|c|c|c|c|c|c|c|}
\hline \multirow{2}{*}{ Variables } & \multicolumn{2}{|c|}{ Sin psicofármacos } & \multicolumn{2}{|c|}{ Con psicofármacos } & \multicolumn{2}{|c|}{ Total } & \multirow{2}{*}{$p$} \\
\hline & $\mathbf{n}$ & $\%$ & $\mathbf{n}$ & $\%$ & $\mathbf{n}$ & $\%$ & \\
\hline \multicolumn{8}{|l|}{ ESTRÉS } \\
\hline No & 41 & 97.6 & 7 & 77.8 & 48 & 94.1 & 0,022 \\
\hline SI & 1 & 2.4 & 2 & 22.2 & 3 & 5.9 & \\
\hline \multicolumn{8}{|l|}{ INSOMNIO } \\
\hline No & 29 & 100 & 19 & 86.4 & 48 & 94.1 & 0,040 \\
\hline SI & 0 & 0 & 3 & 13.6 & 3 & 5.9 & \\
\hline
\end{tabular}

Finalmente, se encontró relación entre la ansiedad de los enfermeros que trabajan en el área COVID-19 y la práctica de alguna religión, con quién conviven en el hogar y el haber sido diagnóstico personalmente positivo a COVID-19 en algún momento de la pandemia. 
Tabla 4. Enfermeros del área COVID-19 y su relación con algunas variables, Hospital Huaraz-Perú.

\begin{tabular}{lllllllll}
\hline \multirow{2}{*}{ Variables } & \multicolumn{2}{c}{ Sin ansiedad } & \multicolumn{2}{l}{ Con ansiedad } & \multicolumn{3}{c}{ Total } \\
& & $\mathbf{n}$ & $\mathbf{\%}$ & & $\mathbf{n} \%$ & $\mathbf{n}$ & $\mathbf{2}$ & $\boldsymbol{P}$ \\
\hline Practicante & $\mathrm{Si}$ & 12 & 38.7 & 4 & 14.3 & 16 & 27.1 & 0.035 \\
religioso & No & 19 & 61.3 & 24 & 85.7 & 43 & 72.9 & \\
& Total & 31 & 100.0 & 28 & 100.0 & 59 & 100.0 & \\
Convivencia & Solo/a & 9 & 29.0 & 2 & 7.1 & 11 & 18.6 & 0.031 \\
& Familia & 22 & 71.0 & 26 & 92.9 & 48 & 81.4 & \\
& Total & 31 & 100.0 & 28 & 100.0 & 59 & 100.0 & \\
Diagnosticado & No & 25 & 80.6 & 15 & 53.6 & 40 & 67.8 & 0.026 \\
con COVID-19 & Si & 6 & 19.4 & 13 & 46.4 & 19 & 32.2 & \\
& Total & 31 & 100.0 & 28 & 100.0 & 59 & 100.0 & \\
\hline
\end{tabular}

\section{DISCUSIÓN}

$\mathrm{R}$ especto a las características de la población, la mayoría de los profesionales de enfermería que trabajan en el área COVID-19 del Hospital Víctor Ramos Guardia de Huaraz son mujeres. Históricamente la profesión de enfermería es propia de las mujeres; asociándose en muy diversas sociedades y culturas a lo largo del tiempo al acto de cuidar con el género femenino. Podemos decir que la influencia del género en la profesión enfermero, ha provocado una falta de reconocimiento social de la misma, al estar asociado de forma simbólica los cuidados enfermeros a las cualidades intrínsecamente femeninas.

El mayor porcentaje de enfermeros que trabaja en el área COVID-19 viven con su familia, la mayoría de estos profesionales laboran menos de 10 años y tienen miedo a contagiar con el COVID-19 a sus familiares; porque el estar en contacto directo con los pacientes COVID19 y ser el contagio directo de persona a persona los hace susceptibles de llevar el virus a su hogar.
Más de la mitad de enfermeros que trabajan en el área COVID-19 manifiestan que trabajan con equipos de protección personal (EPP) incompletos, probablemente por logística insuficiente en la dotación de materiales. Estos datos son semejantes a los hallados en China (14) donde hallaron que una de las razones de la angustia psicológica del personal de salud era que los trabajadores sentían no estar seguros en su trabajo por la escasez de equipos de protección y por lo tanto a la exposición continua a eventos críticos de contagio.

La mayoría de enfermeros que trabajan en el área COVID-19 afirma que alguien en su círculo social fuera de su hogar ha tenido COVID-19, además manifiestan que alguien fuera de su hogar falleció con la COVID-19; datos congruentes con la situación actual de la pandemia en el país por el de aumento masivo de contagios y de la tasa de mortalidad a nivel mundial.

También se aprecia que un tercio de enfermeros que trabajan en el área COVID19 (15) han sido diagnosticado con dicha enfermedad, por pertenecer a un grupo 
profesional que brindan cuidados directos a los pacientes las 24 horas del día y hay un incremento de carga laboral por el aumento de demanda de pacientes infectados en el área de triage, emergencia, consultorio, hospitalización, cuidados intermedios y la unidad de cuidados intensivos.

Un tercio de los enfermeros que laboran en el área COVID-19 tienen insomnio, mientras que casi la mitad del total de enfermeros que trabajan en el área no COVID-19 padecen de insomnio. Estos datos son semejantes a otros estudios (7), donde informaron que el personal de salud que labora en áreas COVID-19 un $34 \%$ presentó de síntomas de insomnio y en otro estudio hallaron $27,8 \%$ de prevalencia de insomnio (16).

En cuanto a la depresión se presentaron casi en un cuarto de los enfermeros que laboran en el área COVID$19 \mathrm{y}$ no COVID-19. Estos datos son semejantes con un estudio realizado en Wuhan (7), donde los trabajadores de la salud informaron síntomas graves de depresión.

Casi la mitad de enfermeros que trabajan en el área COVID-19 tienen ansiedad, y en el área no COVID-19 algo menos; resultados congruentes a estudios donde el 44,6\% de los trabajadores de salud informaron presentar signos de ansiedad y en otro informaron que el $41.3 \%$ de los participantes reportaron síntomas clasificables de moderados a severos de ansiedad $(7,16)$.

En cuanto al estrés podemos decir que el $6,8 \%$ del total de enfermeros que trabajan en el área COVID-19 tienen estrés, mientras que el $17,6 \%$ del total de enfermeros que trabajan en el área no COVID-19 manifiestan estrés. Al parecer la pandemia puede resultar estresante, logrando interferir con la habilidad de vivir una vida normal por un periodo extenso de tiempo (17).

Pero el análisis estadístico determina que en el estudio no hay diferencia significativa entre padecer insomnio, depresión, ansiedad o estrés entre los enfermeros de las áreas COVID-19 y otras áreas diferentes. Estos datos se evidencian frente a la lucha contra el COVID-19, donde los enfermeros enfrentan una enorme presión debido al alto riesgo de infección, debido al incremento de la demanda de trabajo, frustración, cansancio por las largas jornadas laborales $y$ falta de contacto directo con sus familiares; pues el personal de salud puede experimentar problemas de salud mental tales como estrés, ansiedad, síntomas depresivos e insomnio (18).

En la lucha contra la pandemia por COVID-19 los enfermeros se enfrentan diariamente a situaciones que afectan su bienestar y pueden dificultar el trabajo con los pacientes (17). Un ambiente hospitalario congestionado por pacientes con COVID-19 es un entorno complicado de riesgos laborales de salud mental en el trabajador y la gravedad de la situación, va a depender de la exposición, estrategias de afrontamiento e inmunidad de cada persona. De igual modo el estrés y temor de contagiarse con el coronavirus puede ocasionar riesgos psicosociales (19).

Cada persona reacciona de modo distinto ante el impacto psicológico provocado por una epidemia como la COVID-19. El distanciamiento social, la cuarentena y el aislamiento, traen aparejados una serie de reacciones psicológicas que los individuos pueden experimentar en relación a su propio estado de salud (20) En los resultados se puede observar que la medicación 
antidepresiva se relaciona significativamente con el estrés, insomnio, la convivencia con personas mayores de 65 años $y$ el diagnosticado depresivo en enfermeros en el área COVID-19. Asimismo, la ansiedad se relaciona significativamente con la participación en alguna religión, convivencia con familiar y tener diagnóstico con COVID-19.

Estos reportes son similares a los encontrados por Muñoz (21), quien encontró que los trastornos de ansiedad y depresión pueden manifestarse o exacerbarse en situaciones de crisis como la COVID-19, estos están asociados a factores como la capacidad de pedir ayuda, la espiritualidad, tener pareja estable, contar con redes de apoyo como la familia o amigos, tener otras habilidades $\mathrm{y}$ pasatiempos. Asimismo, las relaciones interpersonales, tienen una importancia crítica durante los principales eventos de salud, incluidos la cuarentena y el aislamiento. Estos vínculos deben explorarse y aprovecharse para mejorar los resultados de salud mental. De igual manera, existen circunstancias en la vida de los individuos, que pueden propiciar un mayor riesgo psicosocial ante la pandemia de la COVID-19. Entre estas circunstancias se describen, el ser: Dependiente de algunas sustancias adictivas, Personas con soledad o con la ausencia de redes de apoyo, o presentar ausencia de vínculos con los que mantener una comunicación activa. De igual modo vivir con Menores de edad o sujetos dependientes de otras personas como un adulto mayor (22).

Otro estudio respecto a las principales causas de la ansiedad en la atención a pacientes con COVID-19, se pudo encontrar que oscilan entre el hecho de trabajar en hospitalización de COVID-19, la sospecha permanente de tener COVID-19 en cada día de atención, el que los familiares se hayan infectado con COVID19, ser personal de salud, ser del sexo femenino y consumir algún tipo de medicamento. Barello y otros (23) refieren que los trabajadores sanitarios que trabajaban durante la epidemia COVID-19 informaron de frecuentes preocupaciones con respecto a su propia salud y el temor de infectar a sus familias.

Sin embargo, en un estudio sobre salud mental reporta datos diferentes, pues antes del aislamiento no se veían con frecuencia, no compartían actividades y ahora se sienten mejor en familia, generan mayor empatía y tienen más actividades juntas. El aislamiento social obligatorio ha hecho que las personas se extrañen y se preocupen por sus amigos y familiares que no están con ellos (24).

Con respecto a las variables si ha sido diagnosticado con COVID-19 y fue diagnosticado con alguna enfermedad mental antes de la pandemia, no se ha encontrado estudios al respecto, de allí la importancia de continuar investigando sobre los factores que producen problemas de salud mental en el personal de enfermería a fin de brindar atención preventiva y oportuna.

\section{CONCLUSIONES}

$\mathrm{L}$ a salud mental de los enfermeros no presenta diferencias según el área de trabajo en tiempos de COVID-19. Así mismo como conclusiones complementarias se puede indicar que la depresión de los enfermeros estuvo asociada a la convivencia con personas vulnerables en el hogar y al diagnóstico de enfermedades de salud mental antes de la pandemia por COVID-19. También, la ansiedad de los enfermeros estuvo 
asociada a la convivencia con familiares, a la práctica de alguna religión y al diagnóstico personal de COVID-19.

\section{REFERENCIAS BIBLIOGRÁFICAS}

1. Singhal T. Una revisión de la enfermedad por coronavirus-2019 (COVID-19). Indian J Pediatr. 2020 [Fecha de acceso: 10 de febrero del 2021]; 87 (4): 281-286. Disponible en: https://pubmed.ncbi.nlm.nih.gov/321666 07/

2. Dalglish SL. COVID-19 gives the lie to global health expertise. Lancet. 2020. [Fecha de acceso: 11 de febrero del 2021]. Disponible https://doi.org/10.1016/S01406736(20)30739-X

3. Shen X., Zou X., Zhong X., et al. Estrés psicológico de las enfermeras de la UCI en el momento de COVID-19. Crit Care. 2020; 24(200). [Fecha de acceso: 12 de febrero del 2021]. Disponible en: https://doi.org/10.1186/s13054-02002926-2

4. Muñoz L., Rumie Hossn., Torres G. Impacto en la salud mental de la (del) enfermera(o) que otorga cuidados en situaciones estresantes. Cienc. enferm. 2015;21(1):45-53. [Fecha de acceso: 13 de febrero del 2021]. Disponible en http://dx.doi.org/10.4067/S071795532015000100005

5. Chew N., Lee G., Tan B., et al. Un estudio multinacional y multicéntrico sobre los resultados psicológicos y los síntomas físicos asociados entre los trabajadores de la salud durante el brote de COVID-19. ELSEVIER. 2020. [Fecha de acceso: 14 de febrero del 2021]. Disponible en: https://doi.org/10.1016/j.bbi.2020.04.049

6. Rossi R., Socci V., Pacitti F., et al. Resultados de salud mental entre los trabajadores de atención médica de primera línea y de segunda línea durante la pandemia de la enfermedad por coronavirus 2019 (COVID-19) en Italia.
JAMA Netw Open. 2020;3(5): e2010185. [Fecha de acceso: 15 de febrero del 2021]. Disponible en: http://doi:10.1001/jamanetworkopen.202 0.10185

7. Lai J., Ma S., Wang Y., et al. Los factores asociados con los resultados de salud mental entre los trabajadores sanitarios expuestos a la enfermedad coronavirus 2019. JAMA s Dentro abierto. 2020; 3 (3): e203976. [Fecha de acceso: 16 de febrero del 2021]. Disponible en: https://doi:10.1001/jamanetworkopen.20 20.3976

8. Mason DJ, Friese CR. Proteger a los trabajadores de la salud contra COVID-19 y estar preparados para futuras pandemias. Foro de Salud JAMA. 2020; 1 (3): e200353. [Fecha de acceso: 17 de febrero del 2021]. Disponible en: https://doi:10.1001/jamahealthforum.202 0.0353

9. Naser A., Dahmash E., Rousan R., et al. Estado de salud mental de la población general, profesionales de la salud $y$ estudiantes universitarios durante el brote de la enfermedad por coronavirus de 2019 en Jordania: un estudio transversal. MedRxiv. 2020. [Fecha de acceso: 18 de febrero del 2021]. Disponible en: https://doi.org/10.1101/2020.04.09.2005 6374

10. Gómez E, Fumadó C, Forero C. Impacto psicológico de la cuarentena en los trabajadores de la salud. BMJ journals: Medicina ocupacional y ambiental. [Fecha de acceso: 19 de febrero del 2021]. Disponible en: https://doi:10.1136/oemed-2020-106587

11. Virto C, Virto H, Cornejo A, Loayza W, Álvarez B, Gallegos Y, Triveño J. Estrés ansiedad y depresión con estilos de afrontamiento en enfermeras en contacto con COVID-19 Cusco Perú. Rev. RECIEN. 2020; 9(3):50 - 60. [Fecha de acceso: 20 de febrero del 2021]. Disponible en: https://revista.cep.org.pe/index.php/RECI EN/article/view/44/54 
12. Portocarrero AN, Jiménez-Genchi A. Estudio de validación de la traducción al Español de la Escala Atenas de Insomnio. Salud Ment. 2005;28(5):34-9.

13. Ruiz F, García-Martín M, Suárez J, Odriozola-González P. The hierarchical factor structure of the Spanish version of the Depression Anxiety and Stress Scale 21 (DASS-21). Int J Psychol Psychol Ther [Internet]. 2017;17(1):97-105. Available from:

https://www.researchgate.net/publicatio $\mathrm{n} / 313351122$

14. Zhang W. Mental Health and Psychosocial Problems of Medical Health Workers during the COVID-19 Epidemic in China. Psychother Psychosom 2020;.(89): 242-250. DOI: 10.1159/000507639

15. Organización Mundial de la Salud. (OMS). Preguntas y respuestas sobre la enfermedad por coronavirus (COVID-19). Génova: OPS; 2019

16. Samaniego A. Sintomatología asociada a trastornos de salud mental en trabajadores sanitarios en Paraguay: Efecto COVID-19. Revista Interamericana de Psicología/Interamerican Journal of Psychology 2020;1( 54):1-19.

17. Inchausti F, García-Poveda N, Javier A y Sánchez-Reales S. La Psicología Clínica ante la Pandemia COVID-19 en España. Clínica y Salud 2020; 31(2), 105-107. https://doi.org/10.5093/clysa2020a11

18. Lozano-Vargas A. Impacto de la epidemia del Coronavirus (COVID-19) en la salud mental del personal de salud y en la población general de China. Rev Neuropsiquiatr 2020;83(1): 51-56. https://doi.org/10.20453/rnp.v83i1.3687

19. Soares J, Fernández $M$ y Castelo A. Síndrome del edificio enfermo en tiempos de pandemia por COVID-19. Revista Cubana de Enfermería 2020; 36: e3722
20. Hernández J. Impacto de la COVID-19 sobre la salud mental de las personas. Medicentro Electrónica. 2020. 24(3): 578594

21. Muñoz-Fernández SI, MolinaValdespino D, Ochoa-Palacios R, SánchezGuerrero 0, Esquivel-Acevedo JA. Estrés, respuestas emocionales, factores de riesgo, psicopatología y manejo del personal de salud durante la pandemia por COVID-19. Acta Pediatr Méx 2020; 41 (Supl 1): 127136

22. Asociación de Salud Mental y Psiquiatría Comunitaria. Guía de apoyo psicosocial durante esta epidemia de coronavirus. España: OME-AEN; 2020 [citado 26 marzo 2020]. Disponible en: https://ome-aen.org/guia-de-apoyopsicosocial-durante-esta-epidemia-decoronavirus/

23. Barello S, Falcó-Pegueroles A, Rosa D, Tolotti A, Graffigna G, \& Bonetti L. The psychosocial impact of flu influenza pandemics on healthcare workers and lessons learnt for the COVID-19 emergency: a rapid review. International journal of public health 2020; 65(7): 1205-1216

24. Pacheco E, Bravo D, Pezúa R, Olarte $M$, Espejo K, Rozas V. et al. Memoria del I Encuentro virtual avances y propuestas de investigaciones en salud mental en condiciones de pandemia por COVID-19 realizado los días 02 y 03 de octubre del 2020. 2020; 69 - 83

Conflicto de intereses. Los autores declaran que no existe conflicto de intereses para la publicación del presente artículo científico.

Agradecimiento. Los autores reflejan el esfuerzo y el aporte que las personas aportaron al desarrollo del presente artículo científico. 